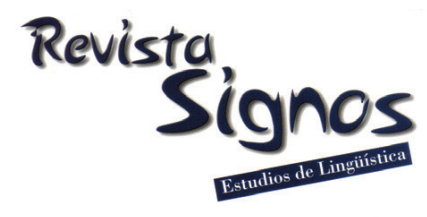

\title{
Organización discursiva de dos géneros en estudiantes de tres niveles de escolaridad y diferentes grupos sociales ${ }^{1}$
}

\section{Discursive organisation of two genres in students from three school levels and different social groups}

\section{Soledad Aravena}

Pontificia Universidad CATÓLICA DE ChILE

CHILE

maravena@uc.cl

\section{Riva Quiroga}

Pontificia Universidad Católica de Chile CHILE

rpquiroga@uc.cl
Javiera Figueroa

PONTIFICIA UNIVERSidad CATÓLICA DE CHILE CHILE jfigueroami@uc.cl

Evelyn Hugo PONTIFICIA UNIVERSIDAD CATÓLICA DE CHILE CHILE eshugo@uc.cl

Recibido: 20/VI/2014 / Aceptado: 23/XII/2015

\section{Resumen}

El presente estudio se propone conocer el efecto del nivel escolar y el contexto social en la organización discursiva de dos géneros escritos. Se analizaron 240 historias personales y artículos explicativos escritos por 120 escolares de tres niveles de escolaridad: Séptimo Básico (12 años), Primero Medio (14 años) y Tercero Medio (16 años) y dos grupos sociales: Medio-Bajo (GSM) y Alto (GSA). Los textos fueron elicitados mediante un breve video sobre los usos de Internet y las redes sociales y luego etiquetados con CLAN. Para el análisis de datos se aplicaron Pruebas T y un diseño de ANOVA factorial mixto con dos factores intergrupales (nivel y grupo social). Los resultados muestran que en el GSM las historias se vuelven menos eventivas y más evaluativas con la edad, pero los artículos explicativos no modifican su organización discursiva. En el GSA hay evaluaciones en todas las edades, pero cambia el uso que se hace de las mismas en textos más extensos y complejos; los artículos explicativos cambian notablemente, tanto en extensión como en organización. Se concluye que la incorporación de nuevos recursos organizacionales y también el uso eficiente de tales recursos para elaborar la información en textos extensos, son características de la trayectoria hacia una producción discursiva competente.

Palabras Clave: Desarrollo, organización discursiva, historia personal, artículo explicativo. 


\begin{abstract}
The study aims to identify the effect of school level and social context in the discursive organization of two written genres. We analysed 240 personal stories and explicative articles written by 120 students from three school levels -7 th grade (12 years old), 9th (14 years olds) and 11th (16 years old) - and two social groups: middle-low socioeconomic status (MSS) and high socioeconomic status (HSS). The texts were elicited by a short video on the uses of Internet and social networks, and then coded with CLAN. T Tests and a design of mixed factorial ANOVA with two intergroup factors (level and social group) were applied for data analysis. The results show that in the MSS group, the stories become less eventive and more evaluative with age/school level, but the explicative texts do not change their discursive organization. In the HSS group, there are evaluation at all school levels, but what evolves is the use of these evaluations in complex and longer texts; the explicative articles change considerably in both length and organization. We conclude that the incorporation of new organization resources and also the efficient use of them to elaborate the information in extended texts, are characteristics of the path to a competent discursive production.
\end{abstract}

Key Words: Development, discursive organisation, personal story, explicative article.

\title{
INTRODUCCIÓN
}

Los desarrollos tardíos del lenguaje (Berman, 2008; Nippold, 2010), propios de la infancia, la adolescencia e incluso de la edad adulta, hacen posible que los niños y jóvenes se conviertan progresivamente en usuarios expertos de su lengua (Berman, 2004). Esto es, que sean capaces de producir discursos extendidos bien formados lingüística, semántica y estructuralmente, con adecuación a diversos contextos y a la vez con una retórica propia y constitutiva de su identidad personal. Estos logros serán cruciales para variados propósitos, que van desde comunicarse eficazmente en su vida cotidiana y participar activamente en su comunidad, hasta la construcción de saberes y la expansión de nuevas formas de razonamiento.

Tales desafíos discursivos, cognitivos y sociales no pueden alcanzarse sin la plataforma del lenguaje escrito y el dominio de sus géneros (Berman \& Ravid, 2009; Tolchinsky, 2009). Por eso resulta importante indagar en el modo en que los niños producen textos escritos y las demandas que esta tarea les impone, considerando los múltiples factores que inciden en su desempeño, tales como la edad, su experiencia letrada y su medio social, entre otros. En este contexto, el objetivo de este estudio es describir y comparar la organización discursiva de producciones escritas correspondientes a dos géneros -una historia personal y un artículo explicativo- en estudiantes chilenos de tres niveles escolares (Séptimo Básico, Primero Medio y Tercero Medio) y dos grupos sociales (Alto y Medio-Bajo). Estos géneros interesan en tanto se consideran ejemplares de una familia o grupo mayor de géneros que comparten rasgos lingüísticos y organizacionales, entre otros (Ciapuscio, 2005): las historias y las explicaciones (Martin \& Rose, 2009). 
Se complementan así hallazgos de trabajos previos sobre la escritura de escolares chilenos que abordan diversos tópicos, entre los cuales se pueden mencionar: proceso de escritura (Marinkovich, 2002); creencias acerca de la escritura (Calderón, 2012); desempeño en la escritura de textos argumentativos (Poblete, 2005) o especializados (Parodi, 2007); coherencia global y otros rasgos en narraciones (Benítez, 2009); coherencia local (Concha, Aravena, Coloma \& Romero, 2010; Concha \& Paratore, 2011); ortografía (Sotomayor, Molina, Bedwell \& Hernández, 2013), entre otros. Muchos de sus resultados contribuyen a un diagnóstico compartido con esta investigación: los estudiantes tendrían un conocimiento impreciso de los requisitos de la comunicación escrita y se enfrentan a la tarea de escritura con escaso dominio tanto de los recursos lingüísticos como de las estrategias asociadas a la escritura narrativa y no narrativa.

El sistema escolar no parece estar en las mejores condiciones para enfrentar este problema. Por una parte, porque se encuentra presionado por el actual contexto de mediciones estandarizadas (Darling-Hammond, 2012), focalizadas casi exclusivamente en la comprensión de textos escritos. Por otra parte, muchos profesores tienen dificultades para lograr una enseñanza efectiva de la escritura por diversas razones: débil formación inicial en este ámbito y falta de actualización (Cox, Meckes \& Bascopé, 2010; Sotomayor, Parodi, Coloma, Ibáñez \& Cavada, 2011), gran número de alumnos por sala, pocas horas disponibles para planificar y corregir, entre otras (Bellei, Valenzuela \& Sevilla, 2009).

Estos factores, sin embargo, afectan de manera distinta a las escuelas según su tipo de dependencia y el grupo socioeconómico al que pertenezcan sus estudiantes. En este sentido, el presente trabajo puede ofrecer información que contribuya a diseñar mejores estrategias de enseñanza para incrementar la práctica y la calidad de la escritura en los grupos considerados.

\section{Marco teórico}

\subsection{Géneros discursivos y desarrollo}

Debido a la naturaleza aplicada de nuestro trabajo, nos interesa la noción de género discursivo, porque los géneros son el resultado de la actividad social y constituyen herramientas que permiten a las personas llevar a cabo acciones lingüísticas y participar en diferentes actividades sociales (Bawarshi \& Reiff, 2010; Prior, 2009). En segundo lugar, porque los conocimientos sobre los géneros resultan fundamentales en la comprensión de los procesos de adquisición de lenguaje oral y escrito y los procesos de enseñanza, tanto de lengua materna como de segundas lenguas (Ciapuscio, 2005; Christie, 2012; Rose \& Martin, 2012). Por último, es necesaria y pertinente la noción de género también debido a su dimensión cognitiva, en el sentido de lo expuesto por Parodi (2008) sobre la relación entre lenguaje, género

y cognición. Es así que "ese conocimiento, elaborado a través de procesos 
ontogenéticos, se almacena en la memoria de los lectores/escritores y hablantes/oyentes en un formato representacional complejo" y que los géneros "descansan y se construyen y reconstruyen, por medio de constructos cognitivos y lingüísticos que, todos juntos, se articulan complejamente” (Parodi, 2008: 27-30).

Durante las últimas décadas, numerosos estudios han revelado que los niños son capaces de distinguir regularidades propias de los géneros desde muy temprano (Alamargot \& Fayol, 2009; Bamberg \& Moissinac, 2003) y que su conocimiento de los mismos evoluciona sustantivamente durante toda la infancia y en los años posteriores. Este desarrollo de los géneros se puede relacionar con formas de razonamiento características de las etapas del desarrollo cognitivo (Donovan \& Smolkin, 2006; Tolchinsky, 2004) y que operan en las diversas dimensiones de los textos, tanto del plano microestructural (opciones léxicas y construcción sintáctica) como del plano macroestructural (organización global).

Una de estas dimensiones es la expresión del posicionamiento (discourse stance; Gray \& Biber, 2012; Reilly, Zamora \& MacGivern, 2005), y sus tres tipos de actitud epistémica, deóntica y afectiva- relacionadas evolutivamente con el dominio de los géneros. La actitud epistémica (que expresa grados de certeza o posibilidad) es un rasgo típico de los hablantes/escritores maduros y del lenguaje académico. No obstante, la investigación señala también como un logro crucial del discurso adulto la habilidad para combinar las tres actitudes de manera flexible y adecuada en un contexto discursivo específico (Berman, Ragnarsdóttir \& Strömqvist, 2002; Ucelli, Dobbs \& Scott, 2013). Otra dimensión importante es la organización discursiva que, por su carácter central en este estudio, se aborda con detención en los apartados siguientes.

\subsection{Organización narrativa: Definiciones básicas y aspectos evolutivos}

Las narraciones han sido definidas como un método de recapitular experiencias pasadas combinando las secuencias verbales de cláusulas con las secuencias de eventos que, según se infiere, en realidad ocurrieron (Labov, 1972). Hay cierto consenso en que la organización básica, común a la familia de géneros narrativos distingue, al menos de manera general, un inicio (evento inicial y/o escenario), un desarrollo (evento complicado, reacción) y un final (resultado, desenlace). Específicamente, el género elicitado en este estudio es una 'historia personal', cuya organización fue adaptada de la propuesta de Labov (1972) en respuesta a las características de la tarea solicitada a los estudiantes. Dicha organización distingue las siguientes fases o componentes: orientación (ubicación temporoespacial e introducción de los personajes), desarrollo de los eventos (que incluye la complicación o evento clave y otros eventos que hacen avanzar la historia), resolución (desenlace) y evaluación (reflexiones del narrador que pueden ser valoraciones generales, recomendaciones, 
explicaciones de los estados mentales propios y ajenos o reflexiones al cierre de la historia). Los eventos y la resolución constituyen el esqueleto eventivo de la historia (foreground o primer plano). En cambio, la orientación y la evaluación son su trasfondo (background o segundo plano) (Hopper, 1979; Labov, 1972).

Estudios evolutivos (en otras lenguas y en español peninsular) muestran que desde edades muy tempranas los niños incluyen el esqueleto eventivo -la secuencia de eventos o primer plano- en sus relatos. En general, elaboran poco la información de los personajes y mantienen en el texto el orden en el cual se producen los sucesos, es decir, construyen sus historias a partir de la enunciación de los eventos (Aravena, 2011; Tolchinsky, 2007), probablemente producto de su activación lineal desde la memoria episódica (Sanders \& Schilperoord, 2006).

Este proceso de construcción del texto ha sido denominado por algunos autores como de tipo ascendente (bottom-up), o dirigido por el estímulo (Berman, 2008; Karmiloff-Smith, 1985). Como resultado, las historias infantiles -desde los 6 a los 10 años- tienden a ser principalmente eventivas. Gradualmente y durante la adolescencia, dicho proceso se transformaría en otro más complejo, capaz de integrar procesos descendentes (top-down) hacia los 16 años, dirigidos más bien por el conocimiento del género y las reflexiones del sujeto en torno a los personajes y los sucesos (Berman, 2008).

Habría entonces en este periodo un cambio decisivo en el modo de contar una historia (Berman \& Nir, 2007; Tolchinsky, 2007): mientras los menores lo entienden como relatar una sucesión de eventos a medida que estos son activados en la memoria, los mayores lo conciben como su propia reflexión sobre los eventos y las personas. Orientados por sus propias reflexiones, sus textos serían más divergentes, tanto respecto de las características del género, como respecto de los textos de sus pares. Con la edad los relatos se tornarían menos eventivos, más reflexivos y más diversos al interior de un mismo grupo. Así, el presente estudio se propuso explorar algunos de estos hallazgos en estudiantes chilenos.

\subsection{Organización explicativa: Definiciones básicas y aspectos evolutivos}

Las explicaciones se caracterizan por generalizar, ya sea describiendo los fenómenos, explicando cómo ocurren los procesos, instruyendo acerca de cómo observar el objeto y reportando/interpretando los resultados de una observación (Martin \& Rose, 2009). Su estudio evolutivo es relevante porque se trata de los textos que más se leen durante los años escolares (Scott, 2010; Shleppegrell, 2004) y tienen un rol fundamental en la transmisión de los saberes académicos y disciplinares (Snow \& Uccelli, 2009). 
La organización de estos discursos varía sustancialmente según su propósito (informativo, procedimental, comparativo, etc.), pero de modo general, incluye: una introducción en la cual se define, clasifica y describe el fenómeno, una secuencia explicativa que elabora, explicando cómo y por qué, y un cierre o conclusión que presenta una síntesis o interpretación (Katzemberger, 2005; Knapp \& Watkins, 2005).

A diferencia de las historias, que se construyen sobre la base de los eventos, las explicaciones se construyen en torno al tópico, el cual funciona como categoría de jerarquía superior bajo la que se subordina el resto de la información (Berman \& Nir, 2007). Dicha información ha sido analizada frecuentemente distinguiendo dos tipos principales de contenido: las generalizaciones (el contenido nuclear) y las especificaciones (el contenido complementario) (Berman \& Nir, 2007). Estos tipos de información también han sido identificados como movimientos de avance (entregan información nueva y relativamente general); movimientos elaborativos (expanden, amplían y desarrollan la información dada en los movimientos de avance) y movimientos unificadores (de síntesis) (Tolchinsky, Rosado, Aparici \& Perera, 2005).

El género elicitado en el presente estudio se ha denominado 'artículo explicativo' por las características de la tarea solicitada a los estudiantes. Su organización incluye las siguientes fases o componentes: introducción (definición del fenómeno); desarrollo del tema (que incluye descripción de rasgos y/o procedimientos de uso), conclusión (cierre a modo de síntesis o comentario personal) y especificación (reflexiones, ejemplos, recomendaciones). La introducción corresponde principalmente a movimientos de avance; la descripción de rasgos se caracteriza por ambos tipos de movimiento -de avance y elaborativos- y además por exigir amplio dominio del tema; la conclusión contiene los movimientos unificadores o de síntesis y la especificación, principalmente movimientos elaborativos concretos (como ejemplos o recomendaciones).

El perfil evolutivo de las explicaciones ha sido menos estudiado que el de las historias. Sin embargo, existe acuerdo acerca de que su dominio proficiente es más tardío (Nippold \& Scott, 2010; Rose \& Martin, 2012). Entre los 6 y los 9 años, las informaciones y explicaciones de los niños suelen ser meras etiquetas, afirmaciones aisladas, listas de atributos y, a veces, incipiente construcción de párrafos (Donovan, 2001). Desde los 10 años y durante la adolescencia (periodo de interés en este estudio) se ha encontrado que los textos escritos que pretenden informar o explicar suelen tener mayoritariamente introducción y desarrollo, pero que la conclusión es un componente menos logrado, incluso en adultos (Tolchinsky, Johansson \& Zamora, 2002).

De forma análoga a lo expuesto para las historias, también se ha relacionado la construcción de estos textos con determinadas formas de procesamiento cognitivo. Hacia los 10 años, las explicaciones se caracterizarían por la presencia casi exclusiva de 
afirmaciones generales y escasa elaboración de la información, lo que demostraría un procesamiento de tipo descendente (top-down, es decir, inverso al tipo de procesamiento para construir relatos en esta edad). Durante los años posteriores esta construcción incluiría también diversos tipos de información específica y elaborativa, lo que reflejaría la integración de ambos tipos de procesamiento (top-down y bottom-up) (Berman \& Nir, 2007; Katzemberger, 2005).

\section{Metodología}

El presente estudio responde a un diseño cuantitativo, descriptivo y cuasiexperimental. Su objetivo es comprar la organización discursiva de historias personales y artículos explicativos escritos por estudiantes de diferentes edades/niveles escolares y grupos sociales. El primer factor (edad/nivel) se relaciona con la menor o mayor experiencia vital y lingüística de los niños y adolescentes, así como con diversos aspectos de su madurez y su desarrollo cognitivo. El segundo (grupo social) se relaciona con el nivel educativo de las familias de origen y, en el caso de Chile, se vincula estrechamente con el tipo de establecimiento escolar (Valenzuela, Bellei \& de los Ríos, 2014).

\subsection{Selección de la muestra y los participantes}

Tras un análisis de Potencia Estadística (Faul, Erdfelder, Lang \& Buchner, 2007), que estableció un $\mathrm{N}$ muestral mínimo de 108 sujetos, se decidió trabajar con 120 participantes (20 alumnos por cada curso y grupo socioeconómico). Ello garantizaba óptimas posibilidades de detectar significaciones estadísticas asociadas a un tamaño de efecto cercano al típicamente considerado como mediano $(0,25)$.

Se seleccionaron dos colegios de Santiago -uno subvencionado y otro particular pagado- pertenecientes a comunas representativas de dos grupos socioeconómicos (medio-bajo y alto, según el Ministerio de Educación). Ambos colegios son mixtos, imparten los contenidos del curriculum nacional y obtienen puntajes SIMCE cercanos al promedio de su dependencia y comuna. Se intentó aislar al máximo el factor socioeconómico de otras variables en la selección de los colegios. No obstante, fue imposible evitar algunas diferencias que, como se comentará, pueden ser importantes en la interpretación de los resultados: el colegio del grupo medio-bajo es religioso y monolingüe, aunque destina horas a la enseñanza del Inglés. El colegio del grupo alto es laico (con clases de Religión), bilingüe en Inglés e implementa el programa de Bachillerato Internacional (BI) en Enseñanza Media. En cuanto a los niveles escolares, se decidió trabajar con Séptimo Básico, Primero Medio y Tercero Medio porque conforman un rango etario relevante para el desarrollo de habilidades discursivas escritas asociadas al dominio de los géneros (Berman \& Ravid, 2009; Berman, 2008). Además, los niveles inferior y superior pueden informar acerca de conductas de entrada y salida (próxima) de la Enseñanza Media en el sistema escolar chileno. 
Se aplicaron dos tareas de escritura (descritas en el siguiente apartado) a todos los estudiantes de estos niveles en ambos colegios (482) y sus textos se revisaron con dos rúbricas debidamente validadas que permitieron asignarles puntajes de desempeño global. Considerando este puntaje, se excluyeron de la muestra todos los sujetos con rendimiento 'sobre' y 'bajo' las 1,96 unidades de Desviación Estándar, medida utilizada habitualmente para la detección de los casos atípicos. Hechas las exclusiones, se seleccionaron de manera aleatoria los 20 estudiantes de cada nivel y establecimiento (10 hombres y 10 mujeres), cuyos 240 textos conformaron el corpus definitivo.

\subsection{Diseño y aplicación de los instrumentos}

Se diseñó una elicitación semiespontánea mediante material audiovisual acerca del tópico, como es habitual en estudios evolutivos y sobre escritura (Behrens, 2008; Berman \& Verhoeven, 2002; Scott \& Windsor, 2000) y un enunciado e instrucciones pertinentes a cada tarea. El tópico de la escritura, Internet y las redes sociales, había sido seleccionado previamente tras la aplicación de una encuesta de intereses a 700 estudiantes de similares establecimientos y niveles escolares de los de la muestra. A continuación, el contexto y las instrucciones de escritura de la historia (izquierda) y del artículo (derecha):

\begin{abstract}
Historias en la Red
Como viste en el video, cada día vivimos distintas experiencias relacionadas con Internet: conocemos personas o proyectos interesantes, nos informamos sobre actualidad, compramos o vendemos, expresamos y compartimos nuestras ideas $y$ emociones, nos divertimos con música y videos, hacemos denuncias o convocatorias, etc.
\end{abstract}

A continuación te pedimos relatar una historia o anécdota que te haya ocurrido a ti o a algún conocido con el uso de Internet (por ejemplo, un encuentro en una red social o comunidad virtual, un conflicto con alguien, alguna actividad realizada en el colegio, la historia de cómo aprendiste a usar estos medios, la historia de una difusión musical o artística gracias a estos medios, etc.).

Tu texto podría aparecer en una revista digital o Blog donde niños y jóvenes cuentan sus experiencias con Internet.

Es importante contar la historia lo más completa posible. Puedes hacer un borrador si lo deseas y utilizar todas las hojas que necesites.

\section{$\underline{\text { Recursos de la Red }}$}

Como viste en el video, Internet ofrece muchos sitios y recursos para conocer nuevas cosas y aprender sobre lo que nos interesa: nos informamos sobre actualidad en la prensa y en las redes sociales, utilizamos buscadores de información y enciclopedias, aprendemos nuevos juegos, creamos y desarrollamos perfiles personales o grupales, compartimos información, música, películas y programas, entre otras posibilidades.

Elige uno de esos sitios o recursos de Internet que te guste y conozcas bien (no importa si no aparece en el video). Una vez elegido, te pedimos escribir un artículo en que lo presentes y lo expliques (por ejemplo, qué es, para qué sirve, cómo se usa, etc.), para personas que no lo conocen y quieren aprender a usarlo.

Tu texto podría aparecer en una revista digital o Blog donde niños y jóvenes aprenden acerca de los usos de Internet.

Es importante informar y explicar de la manera más completa posible. Puedes hacer un borrador si lo deseas y utilizar todas las hojas que necesites. 
El material audiovisual y las tareas de escritura fueron evaluados por cinco expertos en escritura, a quienes se les pidió revisarlos en relación con aspectos tales como pertinencia e interés del tema, claridad de instrucciones, suficiencia de la situación retórica, entre otros. En general los expertos consultados consideraron el material adecuado, pertinente y formulado correctamente, pero sus sugerencias condujeron a una formulación más completa del contexto de las tareas (situación retórica) y a una redacción más clara de las instrucciones. Estas fueron probadas luego en una aplicación piloto $y$, tras leves modificaciones, finalmente aplicadas a los estudiantes en dos instancias de 90 minutos cada una y separadas por un lapso de tres semanas.

\subsection{Transcripción, características del corpus y etiquetado de datos}

Los 240 textos fueron transcritos y segmentados en cláusulas (siguiendo la definición de Berman \& Slobin, 1994). Esta unidad se utilizó como medida para conocer la extensión de los textos y la distribución proporcional de sus componentes (además, las cláusulas eran necesarias para otros análisis - de complejidad sintácticade un proyecto mayor en que se inserta el presente estudio). Los cálculos proporcionales mencionados fueron especialmente necesarios debido a las grandes diferencias en la extensión de los textos, tanto por edad como por género. En la Tabla 1 se presenta la extensión de los textos por edad, grupo y género discursivo:

Tabla 1. Extensión de los textos en $n^{0}$ de cláusulas por grupo y género (promedio y DE).

\begin{tabular}{|c|c|c|c|c|c|c|c|c|}
\hline & \multicolumn{3}{|c|}{ Grupo Medio-Bajo (GSM) } & \multicolumn{3}{c|}{ Grupo Alto (GSA) } \\
\hline \multirow{3}{*}{ Historia } & $\begin{array}{c}\text { Séptimo } \\
\text { (SEP) }\end{array}$ & $\begin{array}{c}\text { Primero } \\
\text { (PRI) }\end{array}$ & $\begin{array}{c}\text { Tercero } \\
(\text { TER })\end{array}$ & Total & $\begin{array}{c}\text { Séptimo } \\
\text { (SEP) }\end{array}$ & $\begin{array}{c}\text { Primero } \\
\text { (PRI) }\end{array}$ & $\begin{array}{c}\text { Tercero } \\
(\text { TER) }\end{array}$ & Total \\
\cline { 2 - 10 } & 43,10 & 36,10 & 54,35 & 44,52 & 33,50 & 54,35 & 75,30 & 54,38 \\
\hline \multirow{2}{*}{ Artículo } & $(19,36)$ & $(19,89)$ & $(32,14)$ & $(25,26)$ & $(7,82)$ & $(19,79)$ & $(28,45)$ & $(26,52)$ \\
\cline { 2 - 10 } & 27,30 & 26,70 & 38,85 & 30,95 & 35,80 & 44,80 & 60,10 & 46,90 \\
\hline
\end{tabular}

Posteriormente, los textos fueron etiquetados con CLAN (MacWhinney, 2014), proceso que consistió en reunir grupos de cláusulas en 'paquetes' de información identificados con los diversos componentes organizacionales de los textos. Dichos componentes fueron preliminarmente definidos a partir de lo señalado en la bibliografía para las características de las tareas elicitadas y luego sometidos a la aprobación de tres expertos en el ámbito del análisis textual y discursivo. Ellos probaron las categorías preliminares en varios textos de la muestra y emitieron sugerencias que permitieron establecer las siguientes categorías definitivas: orientación, complicación, eventos, resolución, evaluación (en las historias personales); e introducción, descripción, procedimiento, conclusión, especificación (en los artículos). Para asegurar un etiquetado consistente, 12 textos fueron etiquetados por cuatro investigadoras del estudio y sus resultados se cotejaron hasta lograr un $100 \%$ de 
acuerdo en los criterios, los que fueron consignados en un exhaustivo manual de etiquetado. Siguiendo estos criterios, dos investigadoras etiquetaron todos los textos, verificando la calibración entre ambas, y una tercera los revisó corroborando la consistencia.

Para el análisis de los datos se aplicó un diseño de ANOVA factorial mixto con dos factores intergrupo. Estos son la edad/nivel escolar (en el entendido de que este factor se asocia con distintos grados de experiencia letrada y lingüística en general) y el grupo socioeconómico (en el entendido de que este factor se relaciona en Chile con el grado de alfabetización familiar y el tipo de colegio y comuna). Este diseño y el tamaño de la muestra permiten hacer afirmaciones generalizables desde el punto de vista estadístico. También se aplicaron pruebas $T$ para muestras independientes con el objetivo de comparar a los estudiantes de un mismo grupo etario y distintos grupos socioeconómicos. En ambos casos, el nivel de significación definido fue de 0,05.

\section{Resultados y discusión}

\subsection{Distribución de componentes de las historias: Comparación por edad y grupo social}

Efecto de la edad. Los resultados muestran el efecto significativo de la edad/nivel escolar en los componentes clave de las historias personales (eventos y evaluación), pero solo en uno de los dos grupos, el GSM. Como muestra la Tabla 2, la proporción de cláusulas que los estudiantes destinan a los eventos disminuye significativamente con la edad $(F(2,57)=5,70, p .=, 006)$ y la que destinan a la evaluación aumenta $(F(2,57)=7,16, p .=, 002)$.

Tabla 2. Cláusulas destinadas a cada uno de los componentes de las historias. Porcentajes sobre el total de número de cláusulas del texto.

\begin{tabular}{|c|c|c|c|c|c|c|}
\hline & \multicolumn{3}{|c|}{ Grupo Medio-Bajo (GSM) } & \multicolumn{3}{c|}{ Grupo Alto (GSA) } \\
\hline & SEP & PRI & TER & SEP & PRI & TER \\
\hline Orientación & 5,5 & 11,6 & 10,3 & 13,3 & 13,7 & 15,2 \\
\hline Complicación & 12,0 & 6,6 & 11,8 & 13,9 & 13,3 & 14,4 \\
\hline Eventos & 49,9 & 50,6 & 28,5 & 31,0 & 38,5 & 26,3 \\
\hline Resolución & 8,3 & 4,1 & 5,1 & 15,5 & 10,2 & 9,9 \\
\hline Evaluación & 11,5 & 16,4 & 33,7 & 25,0 & 16,2 & 26,8 \\
\hline Otro & 12,9 & 10,7 & 10,7 & 1,4 & 8,1 & 7,4 \\
\hline
\end{tabular}

En otras palabras, los estudiantes del GSM muestran cambios en el uso de los componentes de la organización narrativa entre los 12 y los 16 años, y los cambios se ajustan a la trayectoria esperada desde una historia más infantil a otra más adulta: disminuye la presencia eventiva o de primer plano, y aumenta la evaluativa, o de segundo plano. Este resultado confirma lo encontrado por estudios con estudiantes de otros países (Tolchinsky, 2007) según los cuales las historias de los niños pequeños se procesan de manera lineal y ascendente a partir de los eventos, y que luego, en la 
adolescencia, revelan un procesamiento de tipo descendente, regido por una estrategia discursiva.

Los estudiantes del GSA, en cambio, no modifican significativamente el uso que hacen de la organización propia de las historias entre estas edades. Es decir, parecen alcanzar a los 12 años una distribución de componentes organizacionales con fuerte presencia evaluativa -compuesta de valoraciones generales principalmente-. Sin embargo, puesto que sus textos son muy breves y poco elaborados, en ocasiones descuidan los elementos propiamente narrativos para enfocarse en las reflexiones. En suma, los recursos evaluativos ya están presentes en los menores del GSA, que tienden a priorizar las valoraciones por sobre los eventos, al igual que los estudiantes mayores (ejemplos 3 y 4, más abajo), pero sin la productividad verbal ni el dominio lingüístico y discursivo de estos. Como resultado, sus historias pierden fuerza narrativa, como se ilustra en el ejemplo 2.

También se observan diferencias en los tipos de evaluaciones. Mientras que los menores evalúan para hacer afirmaciones generales y taxativas (como puede observarse en el ejemplo 2), los mayores evalúan desde la subjetividad, incorporando matices y perspectivas (ejemplo 4). Se observa una evolución esperable, por lo tanto, desde una actitud de carácter deóntico (típicamente infantil, producto en parte de la internalización de las normas durante la infancia) hacia una actitud epistémica, habitual en los textos académicos y propia de hablantes maduros (Berman et al., 2002).

Efecto del GS. El factor grupo socioeconómico/colegio es significativo solo entre los más jóvenes de ambos colegios, para los componentes de orientación, eventos y evaluación. Así, los estudiantes de SEP del GSA destinan una proporción de cláusulas significativamente mayor en la orientación $(t(38)=-2,31, p .=, 026)$ y también en la evaluación $(t(38)=-2,39, p .=, 021)$, ambos componentes del segundo plano; en cambio, estos estudiantes destinan un número significativamente menor de cláusulas a los eventos $(t(38)=2,63, p .=, 012)$, en relación con sus pares del otro colegio. Es decir, para la distribución de los componentes de las historias, los estudiantes mayores de ambos colegios se comportan de modo similar y según lo esperado, pero los estudiantes menores (SEP) lo hacen de modo diferente: los del GSM construyen historias típicas fundamentalmente centradas en los eventos (ejemplo 1).

(1) Grupo Medio-Bajo, Séptimo.

El chat y el virus

A mí me ha pasado que un día en mis vacaciones yo prendí mi computador, lo inicié, etc. Entré a la página Google.com y luego ya cargada la página puse Buenamusica.com y me puse a escuchar música. Estuve como media hora estando ahí escuchando música. Bueno y vi que había un chat y me puse a hablar.

y luego me empezaron a mandar cuestiones de adultos y otras cosas. La cosa que después me quería salir de la página y la cuestión que no podía. Después llamé a mis papás para que me ayudaran y no se pudo y me dijeron que tenía un virus. 
Después de ver que no tenía un antivirus fuimos adonde un vecino para que lo (eventos) pudiera arreglar. Estuvimos como cuatro horas esperando que lo arreglara. Al final lo arregló. Mi papá preguntó cuánto costaba el arreglo y lo que cobraba era de cincuenta y cinco mil pesos y yo le dije que de dónde saco cincuenta y cinco mil pesos y me dijo no sé, pero me tienen que pagar. Mi papá le pagó con un cheque.

La cosa es que llegamos a mi casa y por supuesto yo pensaba que me iban a castigar pero me dijeron que esto le pasaba a cualquiera y que para la próxima tenga más cuidado y que no ande en cualquier página.

Desde ese día que tengo más cuidado con Internet.

(evaluación$\operatorname{cod} a)$

\section{(2) Grupo Alto, Séptimo.}

Una tragedia entre amigos

Había una vez un amigo mío que fue hackeado por uno de sus mejores amigos... que

(eventos) él sabía su contraseña y usuario. Al final esos dos amigos se detestaron y nunca más fueron amigos.

\begin{tabular}{lc}
\hline $\begin{array}{l}\text { Esto es una tragedia porque quién le haría esto a su mejor amigo. Bueno, eso es lo } \\
\text { que pasa al confiar mucho de alguien y decirle su contraseña y usuario. }\end{array}$ & $\begin{array}{c}\text { (evaluación- } \\
\text { valoración } \\
\text { general) }\end{array}$ \\
\hline $\begin{array}{l}\text { Eso pasa al contarle eso porque podría hablar mal de ti en tu propia sesión de mail o } \\
\text { algo así o podrías terminar como amigo de cualquier persona y esa se hace tu mejor } \\
\text { amigo y te hackea. Así que no confíes en cualquier persona y ten cuidado con quién } \\
\text { hablas y se hace tu amigo, podría terminar hackeándote. }\end{array}$ \\
\hline
\end{tabular}

\section{(3) Grupo Medio-Bajo, Tercero.}

Historia

Es impresionante la cantidad de acciones que puedes realizar con solo un click. Facebook, la red social que a mi parecer es la más masiva en la población actual, es una plataforma en la cual puedes hacer de todo, comunicarte con amigos, con familia, hasta puedes chatear con desconocidos, hacer eventos fiestas, cumpleaños, etc.

Precisamente en esta red social antes mencionada, conocí a mi novia, y todo comenzó por una "solicitud de amistad", hablando virtualmente y gracias a esto, conocernos en persona. Afortunadamente asistimos al mismo colegio, y todo comenzó por saludos en los recreos, y así conocernos más y más, lo cual se lo debo a Facebook.

\begin{tabular}{lc}
\hline Hoy en día estamos en una relación, y llevamos 1 año. & (resolución) \\
\hline Facebook es muchas veces una buena página para armar conflictos, ya sea por ciertos & (evaluación- \\
"comentarios", "estados", y las infaltables fotos, que de cierta forma reflejan tu forma & valoración \\
de ser y personalidad, muchas veces reflejan identidad. por esto y muchos otros & general)
\end{tabular}
motivos esta red social y muchas más hoy en día son importantes, muchas veces reflejan lo cotidiano, y para algunos una forma de vida.

\section{(4) Grupo Alto, Tercero}

Internet hoy en día, resulta ser parte de nuestras vidas.

(evaluaciónvaloración gral.)

\begin{tabular}{lc}
\hline $\begin{array}{l}\text { Mi historia sería completamente distinta si durante mi desarrollo no hubiera existido } \\
\text { este medio de comunicación, difusión y opinión (entre otras definiciones) }\end{array}$ & $\begin{array}{c}\text { (evaluación- } \\
\text { explicación } \\
\text { personal) }\end{array}$ \\
\hline y se da a notar su importante rol, cuando al conversar con alguien, incluimos como & (evaluación- \\
\hline
\end{tabular}




\begin{tabular}{|c|c|}
\hline $\begin{array}{l}\text { "algo que le teníamos que contar", a un hecho que ocurrió; al usar Internet, e Internet } \\
\text { o por él. }\end{array}$ & valoración gral.) \\
\hline El año pasado, me tocó observar una pelea. & (complicación) \\
\hline $\begin{array}{l}\text { Esta vez, no dentro de una película, ni cara a cara, sino de la "forma de los } \\
\text { vergonzosos", en donde pasas a transformarte a un objeto digital que mágicamente te } \\
\text { entrega poderes y valentía. Quizá porque de alguna forma, te enmascara, o porque } \\
\text { escribir en esa pantalla (haciendo referencia al monitor) es como hablar contigo } \\
\text { mismo, porque no está la presencia de tu "adversario". Y es así, como quizá lo usas } \\
\text { como tu diario personal, sin procesar que posiblemente, tu pensamiento y mensaje no } \\
\text { serán tan privados, aunque tengas solamente diez amigos en Facebook. }\end{array}$ & $\begin{array}{l}\text { (evaluación } \\
\text { explicación } \\
\text { personal) }\end{array}$ \\
\hline $\begin{array}{l}\text { La pelea que observé se produjo a la luz pública, e incluso yo me enteré, que fue } \\
\text { porque un "personaje virtual", le robó una idea a otro "personaje virtual", lo que } \\
\text { generó que varios "personajes virtuales" se involucraran en el asunto, defendiendo al } \\
\text { uno o al otro. }\end{array}$ & (eventos) \\
\hline $\begin{array}{l}\text { Parecía un partido de fútbol, en donde observabas el "juego" y a la vez te hacías } \\
\text { partícipe alentando o defendiendo a alguno. }\end{array}$ & $\begin{array}{c}\text { (evaluación- } \\
\text { valoración gral.) }\end{array}$ \\
\hline $\begin{array}{l}\text { Un personaje se desconectó y paró la pelea, que se postergó hasta el día siguiente, } \\
\text { cuando una de ellas rendida y sin concretar nada "deterioró" a su "amiga" } \\
\text { eliminándola de Facebook, como juego virtual, }\end{array}$ & (resolución) \\
\hline $\begin{array}{l}\text { Pero al parecer, solamente virtual, porque después las encontré saludándose casi } \\
\text { completamente normal, pero con un poco de incomodidad, como si hubieran sido } \\
\text { rivales en solamente un juego de Play Station. }\end{array}$ & $\begin{array}{c}\text { (evaluación- } \\
\text { coda) }\end{array}$ \\
\hline
\end{tabular}

Los ejemplos anteriores permiten observar algunas de las diferencias atribuibles a la edad/nivel escolar y también debidas al factor social/colegio comentadas antes. El ejemplo 1 (SEP, GSM) es una historia completa sin orientación y con escasa presencia evaluativa, pero con diversos eventos, complicación y resolución. En cambio, el ejemplo 2 (SEP, GSA), muy breve y centrado en las evaluaciones de valoración general, no alcanza a desarrollarse como una historia completa. Esta tendencia hace que los textos de este grupo, a pesar de tener más componentes, tengan una calidad menor que sus pares etarios del otro colegio. Los ejemplos 3 y 4 pertenecen a los estudiantes mayores de ambos colegios y, si se comparan con (1) y (2) permiten constatar los cambios debidos a la edad/nivel escolar, en particular el mayor dominio de una construcción menos eventiva y más evaluativa en los mayores del GSM (3). En los ejemplos del GSA (2) y (4) vemos que ambos tienen una proporción alta de recursos evaluativos. Sin embargo, solo en el de los mayores estos recursos se integran en una organización narrativa completa, porque estos textos exhiben mayor dominio de los recursos y también porque son mucho más extensos.

\subsection{Distribución de componentes de los artículos explicativos: Comparación por edad y grupo social}

Efecto de la edad. Los resultados muestran el efecto significativo de la edad en todos los componentes explicativos (a excepción de la introducción), pero solo en uno de los grupos: en el GSA (a la inversa de lo sucedido con las historias, en que los cambios 
de recursos se producían en el GSM). Como puede observarse en la Tabla 3, la proporción de cláusulas que los estudiantes del GSA destinan a la descripción de rasgos aumenta significativamente $(F(2,57)=5,45, p .=, 007)$; la que utilizan en el procedimiento disminuye $(F(2,57)=3,48, p .=, 037)$, la destinada a la conclusión aumenta $(F(2,57)=9,93, p .<, 001)$ y la empleada en la especificación disminuye $(F(2,57)=4,63, p .=, 014)$. En otras palabras, en el colegio de GSA, la distribución de los componentes explicativos cambia notablemente entre los 12 y los 16 años. Estos cambios, en gran medida, delinean una trayectoria desde un dominio más infantil a otro más maduro del género. Esto, porque aumentan movimientos elaborativos que exigen un alto grado de conocimiento (la descripción de rasgos) y los movimientos unificadores y de síntesis (conclusión), mientras que disminuyen operaciones más concretas (procedimiento y especificaciones).

Tabla 3. Cláusulas destinadas a cada uno de los componentes de los artículos. Porcentajes sobre el total de número de cláusulas del texto.

\begin{tabular}{|c|c|c|c|c|c|c|}
\hline & \multicolumn{3}{|c|}{ Grupo Medio-Bajo (GSM) } & \multicolumn{3}{c|}{ Grupo Alto (GSA) } \\
\hline & SEP & PRI & TER & SEP & PRI & TER \\
\hline Introducción & 20,8 & 24,9 & 19,1 & 12,7 & 14,2 & 14,8 \\
\hline Descripción & 40,1 & 50,0 & 48,1 & 37,5 & 48,8 & 60,3 \\
\hline Procedimiento & 20,4 & 9,5 & 14,3 & 19,7 & 15,0 & 3,9 \\
\hline Conclusión & 5,3 & 4,1 & 7,8 & 1,8 & 5,1 & 11,5 \\
\hline Especificación & 12,5 & 10,9 & 10,3 & 25,9 & 16,7 & 9,5 \\
\hline Otro & 0,9 & 0,6 & 0,3 & 2,4 & 0,2 & 0,0 \\
\hline
\end{tabular}

En cambio, no se registra efecto de la edad entre los estudiantes del GSM para los componentes explicativos. Tanto a los 12 como a los 16 años, escriben textos con una presencia importante de información genérica en movimientos de avance (introducción) y, a la vez, de operaciones concretas, como los procedimientos y las especificaciones. Todo ello en desmedro de los movimientos elaborativos y de síntesis, normalmente considerados como los más complejos en estos textos. Es una organización discursiva relativamente esperable entre los menores según lo encontrado en investigaciones previas, que califican este tipo de explicaciones como típicamente infantiles (Tolchinsky et al., 2005). No obstante, el hecho de que este modo infantil de construir los textos no se modifique después de los 12 años sorprende, más aun si se considera que es durante los años de Enseñanza Media que este tipo de discurso debería enseñarse y utilizarse más.

Efecto del GS. Este factor es significativo en SEP para dos componentes: la introducción y la especificación. En este nivel, los estudiantes del GSM desarrollan más ampliamente la introducción $(t(38)=2,43, p .=, 019)$ y menos la especificación $(t(38)=2,19, p .=, 034)$ que sus pares del GSA. En PRI el efecto del GS también es significativo en la introducción $\left(t(38)=2,40, p_{.}=, 021\right)$ mientras que entre los estudiantes mayores (TER) no se observa efecto significativo del GS. 


\section{(5) Grupo Medio-Bajo, Séptimo}

Facebook

Esto se llama Facebook. Es una red social que sirve para comunicarse con familiares,

(introducción) amigos lejanos, etc. También sirve para jugar juegos que este sitio tiene como Pet society - Cityville - Plantas v/s zombies entre otros, también sirve para crear grupos por ejemplo para el curso, colegio, juntas, cantantes, etc.

Si usted desea crearse un Facebook solo debe ingresar a www.facebook.com y te saldrá "registro". Ahí debes ingresar datos como tu nombre, apellido, fecha de nacimiento, sexo femenino o masculino, y la contraseña que deseas ponerte. Después de completar estos datos se creará tu Facebook.

A medida del tiempo uno va agregando amigos, familiares, compañeros, etc. Puedes subir fotos, crear álbumes con tus fotografías, además puedes publicar tus estados de (descripción de ánimo, unirte a páginas, crear grupos, unirte a grupos, etc. También puedes revisar los rasgos) muros de tus amigos, ponerle me gusta o comentar sus estados, fotografías, etc.

Esta es la red más utilizada por todos.

(procedimientos)

\section{(6) Grupo Alto, Séptimo}

Facebook

Facebook es un sitio web al cual todas las personas del mundo tienen posibilidad de (introducción) entrar y registrarse.

Facebook se usa generalmente para chatear, subir fotos, comentar qué estás haciendo, también para comunicarte con amigos o familiares que no has visto en años. También tiene juegos.

(descripción de rasgos)

Las personas pueden usar Facebook de forma común y corriente pero otras la pueden usar de muy mala forma. Puede ser que lo molesten por el chat, que lo molesten por (especificaciónsus fotos, estas personas le hacen bullying al otro de una forma muy cobarde. Esto generalmente se da en adolescentes que no saben lo que están haciendo, no tienen conciencia. Las personas que lo usan de forma buena, no molestan hablan con el otro de forma amigable, dicen cosas buenas de el otro en sus fotos etc.

Facebook es casi un calendario o agenda para las otras personas ya que uno comenta lo que quiere saber en un grupo o en el inicio y en menos de un minuto lo puedes (descripción de rasgos) saber. Sirve para cuando uno quiere invitar a un amigo, lo publica, que diga que si quiere venir en su muro y llega a tu casa.

\section{(7) Grupo Medio-Bajo, Tercero}

Facebook

Facebook.com es una red social que sirve para comunicarse, subir fotos a la web, (introducción) comentarlas, etcétera. Es una red social dentro de las más populares junto a Twitter.

Para crear una cuenta en Facebook hay que ingresar a la página (procedimiento) http://www.facebook.com. ahí se abrirá una ventana que dirá ingresar, y más abajo dice crear cuenta.

Ojo, que para pertenecer al "mundo Facebook" antes tienen que tener creada una cuenta de correo electrónico. Es el caso de "Hotmail", "Gmail”, etcétera. 
Para crear una cuenta de Facebook hay que colocar datos como nombres, apellidos, fecha de nacimiento, "nickname", intereses, etcétera.

por último para sociabilizar con tus amigos solo tienes que buscarlos en una barrita

que sale en la parte superior de la página, colocas su nombre y aparece. ahí saldrá "agregar a mis amigos".

Es una red social donde puedes conocer gente, compartir fotos o videos, ver lo que (descripción de tus amigos están haciendo, chatear (conversaciones online escritas) y videollamadas.

rasgos)

(8) Grupo Alto, Tercero.

Facebook es una red virtual que se despliega en el mundo virtual. Este sitio web es (introducción) uno de los más visitados alrededor del mundo, lo que permite al usuario tener acceso a una amplia gama de grupos sociales.

Esta red social permite crear un perfil en donde el usuario puede exponer en parte, datos personales, como su domicilio, su lugar de trabajo, su correo electrónico, número telefónico, etcétera. Muchos comparten también fotos, comentarios, organizan eventos, etc. A pesar de parecer una red muy impersonal, pues muchas personas podrían acceder a tu perfil y apreciar tu información personal, el usuario también es capaz de regular o especificar quiénes pueden y quiénes no pueden acceder a la información expuesta en el perfil.

El mayor uso que le da la gente a este espacio virtual es retomar el contacto con antiguos amigos o gente conocida, conocer nuevas personas y formar nuevas relaciones, organizar eventos, promocionar y hacer propagandas, compartir ideas, iniciar debates, y, generalmente unirse a distintos grupos sociales.

Como el acceso a Internet ha ido aumentando, y cada vez más personas han adquirido conocimientos tecnológicos, en Facebook existe una gran cantidad de usuarios, desde niños, adolescentes, jóvenes, adultos y ancianos.

Incluso muchos países del mundo que generalmente se consideran marginados, también forman parte de esta red social, que ya va convirtiéndose en una de las formas más fáciles de conectarse con el propio círculo social de cada uno.

Hemos visto, a lo largo de los años, la evolución de Internet y los diversos programas

(conclusión) que este sitio social ha ofrecido, pero sin duda alguna que las redes sociales a las que nos permite acceder ha sido uno de los más grandes éxitos de esta aplicación.

Los ejemplos 5 y 6 (SEP, ambos colegios) muestran que los menores del GSM parecen distribuir la información de modo más equilibrado en los distintos componentes de la organización explicativa que sus pares del GSA. Sin embargo, tienen una productividad verbal muy baja, lo que hace que sus textos no alcancen a desplegar recursos que les permitan elaborar mejor la información. Respecto de los cambios atribuibles a la edad/nivel escolar, los ejemplos permiten observar resultados opuestos en los dos colegios: en primer lugar, el hecho de que no hay mayores diferencias entre los textos de los menores y de los mayores del GSM (5) y (7). Incluso, en este caso el texto de SEP tiene una organización más completa que el texto de TER (que no tiene conclusión).

En segundo lugar, se observan grandes diferencias entre los artículos explicativos de SEP y TER del GSA (6) y (8): el de los más jóvenes no tiene conclusión y dedica 
tantas cláusulas a la descripción de rasgos como a las valoraciones generales. En cambio, el texto de TER tiene una descripción de rasgos muy extensa y elaborada (que denota un amplio conocimiento sobre el tema), no despliega operaciones concretas como el detalle de procedimientos e incluye una conclusión a modo de cierre. Por lo tanto, se observa en los mayores un uso más eficiente de los mismos recursos en un texto más extenso (como en las historias personales) pero también la incorporación de nuevos recursos (la conclusión).

\section{CONCLUSIONES}

Los resultados del estudio permiten concluir que, entre los 12 y los 16 años, se producen cambios significativos en la organización de los textos y que estos cambios varían según el género discursivo y el contexto social/experiencia escolar de los estudiantes. A partir de los datos se corrobora la presencia de dos tipos de organización discursiva y se aprecian sus características específicas según el género:

1. Organización discursiva básica (desempeño más infantil o inexperto). En las historias, esta organización se caracteriza por tener una mayor presencia del primer plano narrativo (eventos, complicación). En los artículos explicativos, es una organización caracterizada principalmente por los movimientos de avance (en la introducción y en la descripción de rasgos) y por las operaciones concretas (como procedimientos y especificaciones).

2. Organización discursiva predominantemente compleja (desempeño más maduro o experto). En las historias es una organización con mayor presencia del segundo plano (orientación y evaluación). En los artículos explicativos se caracteriza por una mayor presencia de movimientos elaborativos (descripción de rasgos) y movimientos de síntesis (conclusión).

Además, estos diversos recursos propios de una organización más infantil o de una más madura, se combinan con otra característica de los textos analizados: el uso más o menos eficiente de los recursos en textos más o menos elaborados y extensos. Por lo tanto, dos textos pueden tener los mismos tipos de recursos (por ejemplo, gran presencia de componentes evaluativos) pero son diferentes porque utilizan esos recursos de manera distinta. Los textos más extensos normalmente les darán un uso más elaborado y eficiente. La combinación de ambos rasgos permite delinear esquemáticamente cuatro posibilidades: a) recursos básicos en textos breves; b) recursos básicos en textos más elaborados; c) recursos complejos en textos breves; y d) recursos complejos en textos más elaborados. En las siguientes tablas se puede apreciar esquemáticamente cómo se distribuyen los textos de la muestra, según nivel escolar, tipo de colegio y género. 


\begin{tabular}{|c|c|c|c|c|c|}
\hline \multicolumn{2}{|c|}{ Historias personales } \\
\hline \multicolumn{2}{|c|}{} & $\begin{array}{c}\text { Recursos } \\
\text { básicos en } \\
\text { textos breves }\end{array}$ & $\begin{array}{c}\text { Recursos } \\
\text { básicos en } \\
\text { textos más } \\
\text { extensos y } \\
\text { elaborados }\end{array}$ & $\begin{array}{c}\text { Recursos } \\
\text { complejos en } \\
\text { textos breves }\end{array}$ & $\begin{array}{c}\text { Recursos } \\
\text { complejos en } \\
\text { textos más } \\
\text { extensos y } \\
\text { elaborados }\end{array}$ \\
\hline \multirow{3}{*}{ GSA } & TER & & & & \\
\cline { 2 - 6 } & PRI & & & & \\
\cline { 2 - 6 } & SEP & & & & \\
\hline \multirow{3}{*}{ GSM } & TER & & & & \\
\cline { 2 - 6 } & PRI & & & & \\
\cline { 2 - 6 } & SEP & & & & \\
\hline
\end{tabular}

\begin{tabular}{|c|c|c|c|c|c|}
\hline \multicolumn{2}{|c|}{ Artículos explicativos } \\
\hline \multicolumn{2}{|c|}{} & $\begin{array}{c}\text { Recursos } \\
\text { básicos en } \\
\text { textos breves }\end{array}$ & $\begin{array}{c}\text { Recursos } \\
\text { básicos en } \\
\text { textos más } \\
\text { extensos y } \\
\text { elaborados }\end{array}$ & $\begin{array}{c}\text { Recursos } \\
\text { complejos en } \\
\text { textos breves }\end{array}$ & $\begin{array}{c}\text { Recursos } \\
\text { complejos en } \\
\text { textos más } \\
\text { extensos y } \\
\text { elaborados }\end{array}$ \\
\hline \multirow{3}{*}{ GSA } & TER & & & & \\
\cline { 2 - 6 } & PRI & & & & \\
\cline { 2 - 6 } & SEP & & & & \\
\hline \multirow{3}{*}{ GSM } & TER & & & & \\
\cline { 2 - 6 } & PRI & & & & \\
\cline { 2 - 6 } & SEP & & & & \\
\hline
\end{tabular}

Como vemos en la síntesis de las Tablas anteriores, los cambios que surgen en la organización discursiva entre los 12 y los 16 años son distintos en los dos tipos de colegio. Las historias del GSM cambian significativamente (después de PRI) hacia un desempeño maduro. Las del GSA en tanto exhiben más tempranamente estos cambios. Por otro lado, los artículos del GSM muestran una organización discursiva básica en todos los niveles, mientras que las del GSA adquieren gradualmente una organización más experta.

Estas diferencias pueden explicarse, por una parte, a partir del contexto sociocultural de los estudiantes del GSM, provenientes de familias con una escolaridad en ocasiones incompleta y sin estudios universitarios, lo que incide en los desempeños lingüísticos y en el dominio de los géneros discursivos de los estudiantes (Ball, 2006; Verhoeven \& Vermeer, 2006). Por otro lado, la probabilidad de que estos estudiantes tengan menos práctica de escritura escolar sistemática hace que avancen menos con las historias y que no modifiquen sustancialmente su desempeño con los artículos. En cambio, tal como se sugirió en la introducción, los estudiantes del GSA están probablemente enfrentados a la necesidad de escribir más y más frecuentemente, no solo en la asignatura de lenguaje, sino también en otras disciplinas (producto entre, otros factores, del programa de Bachillerato Internacional). 
Los resultados permiten concluir que el desempeño de los escritores más maduros y expertos es el resultado, no solo de la incorporación de nuevos recursos lingüísticos y discursivos, sino también de la habilidad para gestionarlos eficientemente en un discurso extenso y conectado. Así, los estudiantes mayores (de ambos colegios en los textos narrativos y solo del GSA en los textos explicativos) construyen sus textos con nuevos recursos organizacionales y también con un mayor dominio de los mismos en producciones mucho más extensas. Ello hace posible que puedan desplegar funciones específicas y variadas, como explicar exhaustivamente un fenómeno mediante la elaboración de la información o dar un sentido interpretativo a una historia personal, capacidades ausentes en los niños y también en algunos de los adolescentes más jóvenes de este estudio (los del GSM). Mientras que estos consiguen organizar el discurso para poder 'referir' el mundo y 'reproducir' la experiencia o los rasgos concretos en un discurso bien formado, los mayores parecen usar el lenguaje en un sentido más elaborativo y no ya únicamente reproductivo.

Como se ha dicho, estos cambios están relacionados con la mayor experiencia lingüística y letrada de los mayores y su conocimiento de los géneros. En los artículos explicativos (del GSA), esto se traduce en la elaboración de una gran cantidad de información pertinente y relativamente abstracta y la distancia necesaria para intentar concluirla (aunque no siempre con éxito). En las historias (de ambos grupos), implica la toma de perspectiva respecto de lo que se dice, asumiendo puntos de vista y expresando razonamientos que admiten realidades no unívocas sino posibles, alternativas o divergentes. 


\section{REFERENCIAS BIBLIOGRÁFICAS}

Alamargot, D. \& Fayol, M. (2009). Modelling the development of written composition. En R. Beard, D. Myhill, J. Riley \& M. Nystrand, (Eds.). The SAGE Handbook of Writing Composition (pp. 23-47). Londres: SAGE.

Aravena, S. (2011). El desarrollo narrativo a través de la adolescencia: Estructura global de contenido y referencia personal. Revista Signos. Estudios de Lingüistica, 44(77), 215-232.

Ball, A. (2006). Teaching writing in culturally diverse classrooms. En C. MacArthur, S. Graham \& J. Fitzgerald (Eds.), Handbook of Writing Research (pp. 293-310). Nueva York: Guilford Press.

Bamberg, M. \& Moissinac, L. (2003). Discourse development. En A. Graesser, M. Gernsbacher \& S. Goldman (Eds.), Handbook of Discourse Processes (pp. 39-437). Mahwah, NJ: Lawrence Erlbaum.

Bellei, C., Valenzuela, J. P. \& Sevilla, A. (2009). Working conditions and teachers' status in a market oriented educational system. The case of Chile. Ponencia presentada en el 10th UKFIET International Conference on Education and Development: Politics, Policies and Progress. Oxford, Reindo Unido.

Bawarshi, A. \& Reiff, M. (2010). Genre: An introduction to bistory, theory, research, and pedagogy. West Lafayett: Parlor Press and the WAC Clearinghouse.

Behrens, H. (2008). Corpora in language acquisition research. Ámsterdam: John Benjamins.

Benítez, R. (2009). Análisis descriptivo de narraciones escritas por niños y niñas de tercer año básico. Literatura y Lingüística, 20, 103-123.

Berman, R. (2004). Between emergence and mastery: The long developmental route of language acquisition. En R. Berman (Ed.), Language development across childhood and adolescence. (pp. 9-34). Ámsterdam: John Benjamins.

Berman, R. (2008). The psycholinguistics of developing text construction. Journal of Child Language, 35, 735-771.

Berman, R. \& Slobin, D. (1994). Relating events in narrative. A crosslinguistic developmental study. Hillsdale, NJ: Lawrence Erlbaum.

Berman, R. \& Verhoeven, L. (2002). Cross-linguistic perspectives on the development of text-production abilities: Speech and writing. Written Language and Literacy, $5,1-44$.

Berman, R., Ragnarsdóttir, H. \& Strömqvist, S. (2002). Discourse stance. Written Languages and Literacy, 5, 255-290. 
Berman, R. \& Nir, B. (2007). Comparing narrative and expository text construction across adolescence: a developmental paradox. Discourse Processes, 43(2), 79-120.

Berman, R. \& Ravid, D. (2009). Becoming a literate language user. Oral and written text construction across adolescence. En D. Olson \& N. Torrance (Eds.), The Cambridge Handbook of Literacy (pp. 92-111). Cambridge: Cambridge University Press.

Calderón, M. (2012). Intersections between literacy beliefs and written texts produced by 7 to 10 years old Chilean children. Ponencia presentada en the Lancaster University Postgraduate Conference in Linguistics \& Language Teaching, Reino Unido.

Christie, F. (2012). Language education throught the school years: A functional perspective. Malden: Wiley-Blackwell.

Ciapuscio, G. (2005). La noción de género en la Lingüística Sistémico Funcional y en la Lingüística Textual. Revista Signos. Estudios de Lingüística, 38(57), 31-48.

Concha, S., Aravena, S., Coloma, C. \& Romero, V. (2010). Escritura expositiva en tres niveles de escolaridad: Coherencia y dominio de recursos lingüísticos. Literatura y Lingüística, 21, 75-92.

Concha, S. \& Paratore, J. (2011). Local coherence in persuasive writing: An exploration of Chilean students' metalinguistic knowledge, writing process, and writing products. Written Communication, 28(1), 34-69.

Cox, C., Meckes, L. \& Bascopé, M. (2010). La institucionalidad formadora de profesores en Chile en la década del 2000: Velocidad del mercado y parsimonia de las políticas. Pensamiento Educativo, 46/47, 205-45.

Darling-Hammond, L. (2012). Educar con calidad y equidad: Los dilemas del siglo XXI. Santiago: Fundación Chile.

Donovan, C. (2001). Children's development and control of written story and informational genres: Insights from one elementary school. Research in the Teaching of English, 18, 345-366.

Donovan, C. \& Smolkin, L. (2006). Childrens's understanding of genre and writing development. En C. MacArthur, S. Graham \& J. Fitzgerald (Eds.), Handbook of Writing Research (pp. 131-143). Nueva York: Guilford Press.

Faul, F., Erdfelder, E., Lang, A. G. \& Buchner, A. (2007). G*Power 3: A flexible statistical power analysis program for the social, behavioral, and biomedical sciences. Behavior Research Methods, 39, 175-191. 
Gray, B. \& Biber, D. (2012). Current conceptions of stance. En K. Hyland \& C. Sancho (Eds.), Stance and voice in written academic genres (pp. 15-32). Londres: Palgrave.

Hopper, J. (1979). Aspect and foregrounding in discourse. En T. Givón (Ed.), Syntax and Semantics 12: Discourse and Syntax (pp. 213-241). Nueva York: Academic Press.

Karmiloff-Smith, A. (1985). Language and cognitive processes from a developmental perspective. Language and Cognitive Processes, 1(1), 61-85.

Katzemberger, I. (2005). The super-structure of written expository texts -A developmental perspective. En D. Ravid \& H. Shyldkrot (Eds.), Perspectives on language and language development. Essays in honor of Ruth Berman (pp. 327-336). Boston, MA: Kluwer Academic Publishers.

Knapp, P. \& Watkins, M. (2005). Genre, text, gramar. Techonogies for teaching and assessing writing. Sidney: University of New South Wales Press.

Labov, W. (1972). Language in the inner city: Studies in the Black English Vernacular. Philadelphia, PA: University of Pennsylvania Press.

MacWhinney, B. (2014). The CHILDES Project: Tools for analyzing talk. Electronic Edition [en línea]. Disponible en: http://childes.psy.cmu.edu/

Marinkovich, J. (2002). Enfoques de proceso en la producción de textos escritos. Revista Signos. Estudios de Lingüistica, 35(51-52), 217-230.

Martin, J. \& Rose, D. (2009). Genre relations. Mapping culture. Londres: Equinox Publishing.

Nippold, M. (2010). Language sampling with adolescents. San Diego, CA: Plural Publishing.

Nippold, M. \& C. M. Scott, (2010). Expository discourse in children, adolescents, and adults: Development and disorders. Nueva York: Psychology Press/Taylor \& Francis.

Parodi, G. (2007). Comprensión y aprendizaje a partir del discurso especializado escrito: Teoría y empiria. En G. Parodi (Ed.), Lingüistica de Corpus y Discursos Especializados: Puntos de Mira (pp. 223-255). Valparaíso: Ediciones Universitarias de Valparaíso.

Parodi, G. (2008). Géneros discursivos y lengua escrita: Propuesta de una concepción integral desde una perspectiva sociocognitiva. Letras, 51(80), 19-54.

Poblete, C. (2005). Producción de textos argumentativos y metacognición. Letras, 47(71), 63-88. 
Prior, P. (2009). From speech genres to mediated multimodal genre systems: Bakhtin, Voloshinov, and the question of writing. En C. Bazerman, A. Bonini \& D. Figueiredo (Eds.), Genre in a changing world (pp. 17-34). Fort Collins, CO: The WAC Clearinghouse and Parlor Press [en línea]. Disponible en: http://wac.colostate.edu/books/genre/chapter2.pdf

Reilly, J., Zamora, A. \& McGivern, T. (2005). Acquiring perspective in English: The development of stance. Journal of Pragmatics, 37, 185-208.

Rose, D. \& Martin, J. R. (2012). Learning to write, reading to learn. Londres: Equinox.

Sanders, T. \& Schipellroord, J. (2006). Text structure as a window on the cognition of writing: How text analysis provide insights in writing products and writing processes. En C. MacArthur, S. Graham \& J. Fitzgerald (Eds.), Handbook of Writing Research (pp. 386-401). Nueva York: Guilford Press.

Shleppegrell, M. (2004). The language of schooling. A functional linguistics perspective. Mahwah, NJ: Lawrence Erlbaum.

Scott, C. (2010). Assessing expository texts produced by school age children and adolescents. En M. Nippold \& C. Scott (Eds.), Expository discourse in children, adolescents, and adults: Development and disorders (pp. 191-214). Nueva York: Taylor \& Francis Group, Psychology Press.

Scott, C. \& Windsor, J. (2000). General language performance measures in spoken and written narrative and expository discourse of school age children with language learning disabilities. Journal of Speech, Language, and Hearing Research, 43, 324-339.

Snow, C. \& Uccelli, P. (2009). The challenge of academic language. En D. Olson \& N. Torrance (Eds.), The Cambridge Handbook of Literacy (pp. 112-132). Cambridge: Cambridge University Press.

Sotomayor, C., Molina, D., Bedwell, P. \& Hernández, C. (2013). Caracterización de problemas ortográficos recurrentes en alumnos de escuelas municipales chilenas de $3^{\circ}, 5^{\circ}$ y $7^{\circ}$ básico. Revista Signos. Estudios de Lingüistica, 46(81), 105131.

Sotomayor, C., Parodi, G., Coloma, C., Ibáñez, R. \& Cavada, P. (2011). La formación inicial de docentes de Educación General Básica en Chile. Pensamiento Educativo, 48(1), 28-41.

Tolchinsky, L. (2004). The nature and scope of later language development. En R. Berman (Ed.), Language development across childhood and adolescence (pp. 233-247). Ámsterdam: John Benjamins. 
Tolchinsky, L. (2007). El desarrollo de la narración oral y escrita más allá de la infancia. Ponencia presentada en el Coloquio Internacional: Las narrativas y su impacto en el desarrollo infantil, Colegio de México, México DF.

Tolchinsky, L. (2009). The configuration of literacy as a domain of language. En D. Olson \& N. Torrance (Eds.), The Cambridge Handbook of Literacy (pp. 468-486). Cambridge: Cambridge University Press.

Tolchinsky, L., Johansson, V. \& Zamora, A. (2002). Texts opening and closings: Textual autonomy and differenciation. Written Language and Literacy, 5(2), 219254.

Tolchinsky, L., Rosado, E., Aparici, M. \& Perera, J. (2005). Becoming proficient educated users of language. En D. Ravid \& H. Bat-Zeev (Eds.), Perspectives on Language and Language Development. Essays in Honor of Ruth Berman (pp. 375-389). Dordrecht: Kluwer.

Uccelli, P., Dobbs, C. L. \& Scott, J. (2013). Mastering academic language: Organization and Stance in the Persuasive Writing of High School Students. Written Communication, 30(1), 36-62.

Valenzuela, J. P., Bellei, C. \& De Los Ríos, D. (2014). Socioeconomic school segregation in a market-oriented educational system. The case of Chile. Journal of Education Policy, 29(2), 217-241.

Verhoeven, L. \& Vermeer, A. (2006). Sociocultural variation in literacy achievement. British Journal of Educational Studies, 54(2), 189-211.

\section{NOTA}

1 Este estudio ha sido posible gracias al financiamiento del programa FONDECYT al proyecto "Construcción de los discursos narrativo y expositivo escritos en estudiantes chilenos de tres niveles de escolaridad y dos grupos socioeconómicos: Análisis de la estructura global del contenido y la complejidad lingüística" (2011-2014). 